\title{
Monitoring the Evolution of SNR 1987A with Chandra
}

\author{
Sangwook Park, David N. Burrows, Gordon P. Garmire \\ Department of Astronomy and Astrophysics, 525 Davey Lab, \\ Pennsylvania State University, University Park, PA 16802, USA \\ Svetozar A. Zhekov \\ Space Research Institute, Moskovska str. 6, Sofia-1000, Bulgaria \\ Dick McCray \\ JILA, University of Colorado, Box 440, Boulder, CO 80309, USA
}

\begin{abstract}
We report on the results of our monitoring program of SNR 1987A with the Chandra X-Ray Observatory. The high resolution images and the spectra from the latest Chandra data suggest that the blast wave has reached the dense inner ring on the western side of the SNR, as well as in the east. The observed soft X-ray flux is increasing more rapidly than ever, and the latest flux is four times brighter than three years ago.
\end{abstract}

\section{Introduction}

We continue our monitoring program of SNR 1987A with the Advanced CCD Imaging Spectrometer (ACIS) on board the Chandra X-Ray Observatory. As of 2002 December, we have performed a total of seven observations (Table 1). Results from the first six observations have been reported in the literature (Burrows et al. 2000; Park et al. 2002, 2004; Michael et al. 2002). The X-ray morphology was ring-like and asymmetric (brighter in the east) with the emergence of $\mathrm{X}$-ray-bright spots. The X-ray spectrum was described with a plane-parallel shock in a non-equilibrium ionization (NEI) state with an electron temperature of $k T \sim 2.5 \mathrm{keV}$. A blast wave shock velocity of $v \sim 3500 \mathrm{~km} \mathrm{~s}^{-1}$ was derived from the detected X-ray line profiles, which also provided direct evidence of an electron-ion non-equilibrium behind the shock. The X-ray flux was non-linearly increasing as the blast wave approached the dense inner ring. We here report on the results from the latest Chandra observations of SNR 1987A.

\section{Morphology}

The ACIS images (Fig. 1) have been generated by the data reduction process described by Burrows et al. (2000) and Park et al. (2002). Figure 1 shows the overall brightening and development of new X-ray bright spots, particularly on the western side of the SNR. SNR 1987A is expected to be a complete ring in 
Table 1. Chandra ACIS observations of SNR 1987A.

\begin{tabular}{clcc}
\hline ObsiD & Date $\left(\right.$ Age $\left.^{a}\right)$ & Exposure $(\mathrm{ks})$ & Source Counts \\
\hline $124+1387^{b}$ & 1999-10-06 (4609) & 116 & 690 \\
122 & 2000 Jan 17 (4711) & 9 & 607 \\
1967 & 2000 Dec 07 (5038) & 99 & 9031 \\
1044 & 2001 Apr 25 (5176) & 18 & 1800 \\
2831 & 2001 Dec 12 (5407) & 49 & 6226 \\
2832 & 2002 May 15 (5561) & 44 & 6429 \\
3829 & 2002 Dec 31 (5791) & 49 & 9274 \\
\hline
\end{tabular}

a Day since the SN explosion.

$b$ The first observation was split into two sequences, ACIS-S3 and HETG, which were combined in the analysis. The ACIS-S3 configuration was chosen for the other observations.

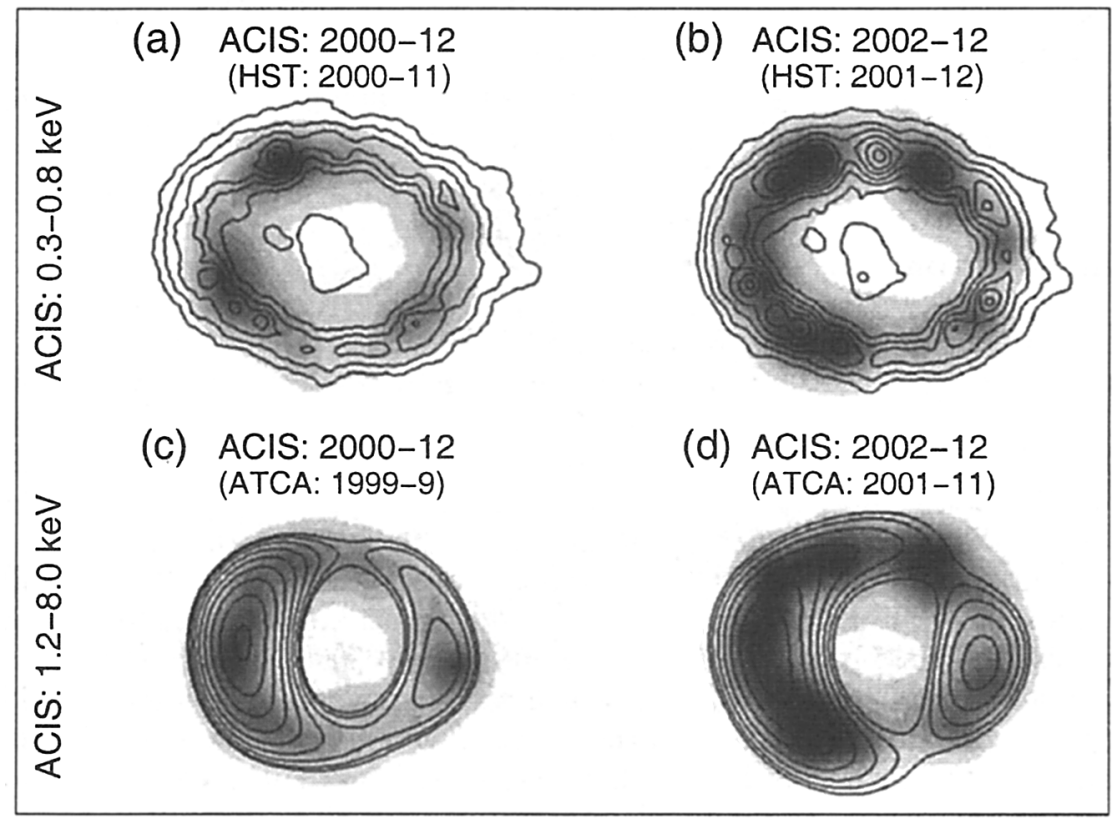

Figure 1. The exposure-corrected ACIS images of SNR 1987A. Darker gray-scales are higher intensities. The image deconvolution and smoothing, after performing sub-pixel resolution imaging, have been applied. Top panels $(\mathrm{a}, \mathrm{b})$ are data in the energy range $0.3-0.8 \mathrm{keV}$, and bottom panels (c, d) show the 1.2-8.0 keV band images. Optical and radio contours are overlaid as indicated. 
(a)

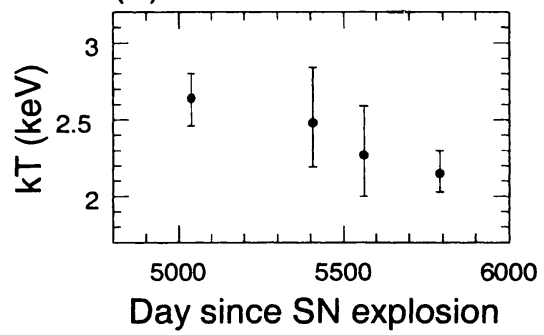

(b)

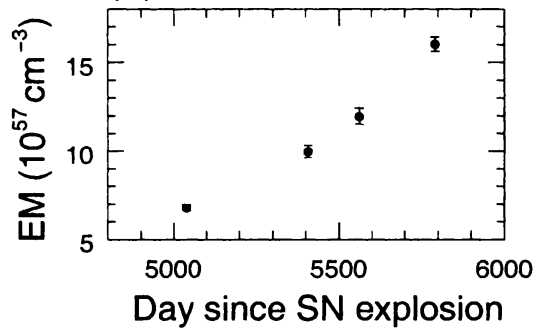

Figure 2. (a) The electron temperature and (b) the emission measure variations of SNR 1987A between 2000 Dec and 2002 Dec.

X-rays. In 2000, the strong correlation between the soft X-ray and the optical images was interpreted as emission from the slow shock encountering protrusions of the dense inner ring. The correlation between the hard X-ray and the radio images was consistent with emission from the fast shock propagating into an H II region interior to the ring. As of 2002 December, correlations between the X-ray and the optical/radio images are more complex than in such a simple picture, which is perhaps expected as the blast wave is now reaching the main body of the inner ring.

\section{Spectrum and Flux}

The X-ray spectrum from each observation can be fitted with a single NEI plane shock model with an electron temperature of $k T \sim 2-3 \mathrm{keV}$ and low abundances (0.1-0.5 solar). Based on the data with the most significant photon statistics, we find that the electron temperature has decreased slightly, while the volume emission measure (EM) has substantially increased for the last two years (Fig. 2). These results are consistent with our physical picture of the blast wave encountering a dense circumstellar medium (CSM). A growing contribution to the observed spectrum from the slow shock is then expected (Michael et al. 2002).

A two-temperature model indeed statistically improves the fit, particularly with the latest data (Fig. 3). The soft component ( $k T \sim 0.25 \mathrm{keV})$ implies a slow shock velocity $\left(v \sim 400 \mathrm{~km} \mathrm{~s}^{-1}\right)$ with a highly advanced ionization state $\left(n_{e} t \sim 10^{13} \mathrm{~cm}^{-3} \mathrm{~s}\right)$. This is consistent with the decelerated shock front in the dense CSM. The $0.5-5 \mathrm{keV}$ band flux from the soft component has tripled since 2000 Dec, while the flux from the hard component has doubled. We note that the flux increase from the slow shock in the western half (by a factor of six) is much larger than in the east $(\sim 30 \%)$. The flux increase from the fast shock is constant between the east and the west. These results suggest that the blast wave has now begun to encounter the dense CSM in the western side of the SNR a few years after it did in the eastern side. The X-ray lightcurve shows a non-linear flux increase rate (Fig. 4). We have thus attempted non-linear fits to the data: e.g., in Figure 4, we present a fit by assuming an exponential density distribution along the radius of the inner ring. The best-fit model implies an 


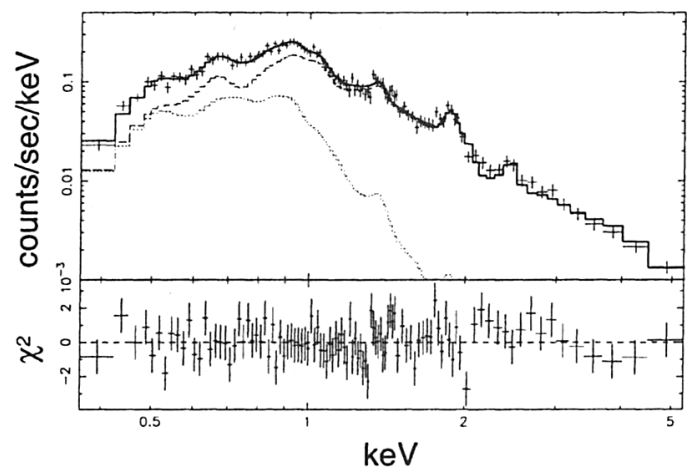

Figure 3. The X-ray spectrum of SNR 1987A as of 2002 Dec. The best-fit two-component plane shock model is overlaid.

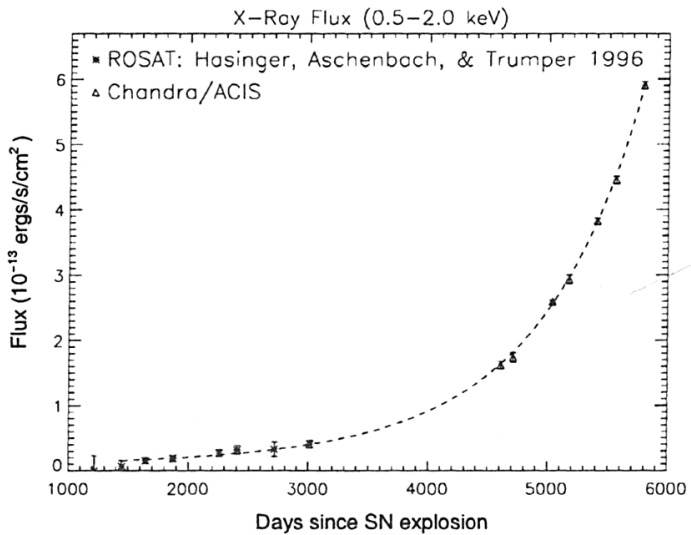

Figure 4. The X-ray lightcurve of SNR 1987A. The dashed curve is a best-fit model assuming an exponential distribution of the radial density profile interior to the inner ring.

$\sim 20$ times higher density in the inner ring than in the HII region. The actual density contrast, which might be higher than this, could be measured as the shock / CSM interaction proceeds.

\section{References}

Burrows, D. N. et al. 2000, ApJ, 543, L149

Michael, E. et al. 2002, ApJ, 574, 166

Park, S. et al. 2002, ApJ, 567, 314

Park, S. et al. 2004, Adv. Sp. Res., 33, 386 